International Journal of Pure and Applied Mathematics

Volume 94 No. 4 2014, 551-559

ISSN: 1311-8080 (printed version); ISSN: 1314-3395 (on-line version)

url: http://www.ijpam.eu

doi: http://dx.doi.org/10.12732/ijpam.v94i4.11

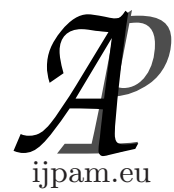

\title{
A SUBDIFFUSION HEAT EQUATION WITH ROBIN CONDITION
}

\author{
A.M. Marín ${ }^{1}$, R.D. Ortíz ${ }^{2}$ \\ J.A. Rodriguez-Ceballos ${ }^{3}$ \\ 1,2,3 Department of Mathematics \\ University of Cartagena \\ Cartagena de Indias, 130015, COLOMBIA \\ ${ }^{3}$ Morelia Institute of Technology \\ Morelia, 58117, MEXICO
}

\begin{abstract}
In this paper we consider a nonhomogeneous subdiffusion heat equation of fractional order with Robin boundary conditions.
\end{abstract}

AMS Subject Classification: 26A33

Key Words: modeling, anomalous diffusion, fractional partial differential equation

\section{Introduction}

In this paper, a nonhomogeneous initial boundary value problem for the time fractional diffusion heat equation in the interval will be studied. This problem was obtained from the nonhomogeneous diffusion heat equation by replacing the first order time derivative by a fractional derivative of order $0<\alpha<1$ in Caputo's sense. In this work, we solve the nonhomogeneous subdiffusion heat equation with fractional time, initial condition and Robin boundary condition. This equation has been recently treated by a number of authors (see Djrbashyan [1] and Kilbas et al.[3]).

Received: April 10, 2014

(C) 2014 Academic Publications, Ltd.

$\S$ Correspondence author url: www.acadpubl.eu 


\section{Preliminary Notions}

In this section, we present some basic definitions and preliminary data that are used throughout the document.

Definition 1. Here we define the following functions for complex argument $z \in \mathbf{C}$. The Mittag-Leffler type functions are defined by:

$$
\begin{gathered}
E_{\alpha}(z):=\sum_{j=0}^{\infty} \frac{z^{j}}{\Gamma(\alpha j+1)}, \\
E_{\alpha, \beta}(z):=\sum_{j=0}^{\infty} \frac{z^{j}}{\Gamma(\alpha j+\beta)}, \\
e_{\alpha}^{\zeta z}:=z^{\alpha-1} E_{\alpha, \alpha}\left(\zeta z^{\alpha}\right),
\end{gathered}
$$

where $\zeta \in \mathbf{C}, \quad \alpha, \beta>0$ and $\Gamma(\cdot)$ is Euler's Gamma function defined for any complex number $z$ as

$$
\Gamma(z):=\int_{0}^{\infty} t^{z-1} e^{-t} d t, \quad z \in \mathbf{C} .
$$

Note that these functions are generalizations of the exponential function base $e$, as $e^{z}=\sum_{j=0}^{\infty} z^{j} / j$ ! and $j !=\Gamma(j+1)$.

Definition 2. If $g(t)$ is a continuous function in the interval $[a, b](g(t) \in$ $\mathrm{C}[a, b])$ and $\alpha>0$, then its Riemann-Liouville fractional integral is defined by

$$
I_{a+}^{\alpha} g(t)=\frac{1}{\Gamma(\alpha)} \int_{a}^{t} \frac{g(s)}{(t-s)^{1-\alpha}} d s .
$$

Definition 3. The Caputo-Djrbashyan fractional derivative of order $\alpha>0$ of a continuous function $g:(a, b) \longrightarrow \mathbf{R}$ is defined by

$$
\left(\frac{d}{d t}\right)^{\alpha} g(t)=I_{0+}^{n-\alpha} g^{(n)}(t),
$$

where $n=[\alpha]+1$, (the notation $[\alpha]$ denotes the largest integer not greater than $\alpha)$.

Lemma 4. (Kilbas et al. [3]). Let $p, q \geq 0$, and $\phi(t)$ be a function of absolute value integrable on an interval $[0, T]$ (namely, $|\phi(t)|$ is integrable on $[0, T]$ or $\left.\phi(t) \in L_{1}[0, T]\right)$. Then

$$
I_{0+}^{p} I_{0+}^{q} \phi(t)=I_{0+}^{p+q} \phi(t)=I_{0+}^{q} I_{0+}^{p} \phi(t)
$$


is satisfied almost everywhere (i.e., except in a set of measure 0) on $[0, T]$. If further $\phi(t)$ is continuous in the interval $(\phi(t) \in \mathrm{C}[0, T])$, then (1) is true and

$$
\left(\frac{d}{d t}\right)^{\alpha} I_{0+}^{\alpha} \phi(t)=\phi(t)
$$

for all $t \in[0, T]$ and $\alpha>0$.

Theorem 5. (Djrbashyan [1]). Let $\phi(t) \in L_{1}[0, T]$. Then, the integral equation

$$
\varphi(t)=\phi(t)+\frac{\gamma}{\Gamma(\alpha)} \int_{0}^{t}(t-\tau)^{\alpha-1} \varphi(\tau) d \tau
$$

has a unique solution $\varphi(t)$ defined by the following formula:

$$
\varphi(t)=\phi(t)+\gamma \int_{0}^{t} e_{\alpha}^{\gamma(t-\tau)} \phi(\tau) d \tau
$$

where $e_{\alpha}^{\gamma z}$ is a Mittag-Leffler type function given in Definition 1.

\section{Technical Development Model}

Let $W(x, t):[0, a] \times[0, \infty) \longrightarrow \mathbf{R}$ be the temperature function at the point $x$ and time $t$. We denote the intensity of heat source at point $x$ and time $t$ by the form $F(x, t)$. The initial temperature (in time $t=0$ ) in this system is denoted by $f(x)$.

Thus, we have a model of anomalous subdiffusion inhomogeneous heat conduction equation with fractional time

$$
\left(\frac{\partial}{\partial t}\right)^{\alpha} W=\kappa \frac{\partial^{2} W}{\partial x^{2}}+F(x, t), \quad 0<x<a, \quad t>0
$$

(the constant $\kappa>0$ is the thermal diffusivity), subject to the boundary condition and initial condition

$$
\begin{gathered}
W(0, t)=0, \quad W_{x}(a, t)=-\beta W(a, t), \quad \beta<0, \quad t>0, \\
W(x, 0)=f(x), \quad 0 \leq x \leq a
\end{gathered}
$$

with the fractional derivative order $\alpha \in(0,1)$ in the sense of Caputo. 
Theorem 6. Let the differential equation (2) satisfy the boundary and initial condition (3). Then the solution of the problem is unique and has the form

$$
\begin{aligned}
W(x, t)= & \sum_{m=1}^{\infty} \mathcal{A}_{m} E_{\alpha}\left(\left[-\kappa \mu_{m}^{2}\right] t^{\alpha}\right) \sin \mu_{m} x \\
& +\sum_{m=1}^{\infty} \mathcal{E}_{m} \int_{0}^{t} e_{\alpha}^{\left[-\kappa \mu_{m}^{2}\right](t-\tau)} f_{k}(\tau) d \tau \sin \mu_{m} x
\end{aligned}
$$

where functions $f_{k}(t)$ are given by (13) for subscript $m$ corresponding, coefficients $\mathcal{E}_{m}$ are given by (11), coefficients $\mathcal{A}_{m}$ are given, for $m \geq 1$, by

$$
\mathcal{A}_{m}=\sqrt{\frac{1}{\int_{0}^{a} \sin ^{2} \mu_{m} x d x}} \int_{0}^{a} f(x) \sin \mu_{m} x d x .
$$

Proof. According to the method of (DuChateau and Zachmann [3]), which we can use due to homogeneous boundary conditions, the solution is sought $W(x, t)$ in the form of the Fourier series of functions $\left\{U_{k}\right\}_{k=1}^{\infty}$ of the linear differential operator $\mathcal{L}$, defined for function $U$ and twice continuously differentiable by the expression

$$
\mathcal{L} U=-\kappa \nabla^{2} U,
$$

where the Laplacian $\nabla^{2}$ in dimension 1 is defined as

$$
\nabla^{2} U \equiv \frac{\partial^{2} U}{\partial x^{2}}
$$

The operator $\mathcal{L}$ is set to some subset of the vector space $\mathbf{L}^{2}[0, a]$ of the functions $U(x), x \in[0, a]$ such that the function $|U(x)|^{2}$ is integrable on $[0, a]$. More precisely, the domain of definition $G_{\mathrm{L}}$ of the operator $\mathcal{L}$ consists of all functions $U(x) \in \mathbf{L}^{2}[0, a]$ satisfying the boundary conditions:

$$
U(0, t)=0, \quad U_{x}(a, t)=-\beta U(a, t), \beta<0 \quad t>0,
$$

and whose images $\mathcal{L} U \in \mathbf{L}^{2}[0, a]$.

The eigenvalue problem is posed as follows. You have to find the values of the parameter $\Lambda$ (eigenvalues of the operator $\mathcal{L}$ ) such that the equation

$$
\mathcal{L} U=\Lambda U
$$


has nontrivial solutions (non-zero) in the domain $G_{\mathrm{L}}$. These functions are the functions of $\mathcal{L}$. Equation (8) is equivalent to the Helmholtz equation

$$
\nabla^{2} U+\frac{\Lambda}{\kappa} U=0
$$

Let $\mu^{2}=\Lambda / \kappa$. So the equation is written

$$
\nabla^{2} U+\mu^{2} U=0
$$

To solve equation (9), we assume a nontrivial solution in the form

$$
U(x)=X(x) .
$$

The corresponding derivatives are:

$$
\begin{gathered}
\frac{\partial U}{\partial x}=X^{\prime}(x), \\
\frac{\partial^{2} U}{\partial x^{2}}=X^{\prime \prime}(x) .
\end{gathered}
$$

Then for equation (9), we have

$$
X^{\prime \prime}(x)+\mu^{2} X(x)=0 .
$$

The solution corresponding to (10) can be expressed as $X(x)=A \cos \mu x+$ $B \sin \mu x$. The boundary conditions imply that

$$
X(0)=0, \quad X_{x}(a)=-\beta X(a),
$$

so we have $A=0$ and

$$
\tan \mu a=-\mu / \beta, \quad B \neq 0 .
$$

The latter gives $0<\mu_{1}<\mu_{2}<\mu_{3}<\cdots, \frac{(2 m-1) \pi}{2 a}<\mu_{m}<\frac{m \pi}{a}, \quad m=$ $1,2, \ldots$ and $\mu_{m} \rightarrow \frac{(2 m-1) \pi}{2 a}$ as $m \rightarrow \infty$. Accordingly,

$$
X_{m}(x)=B_{m} \sin \mu_{m} x, m=1,2, \ldots
$$

The solutions of equation (9) can be written as

$$
\begin{aligned}
U_{m}(x) & =\mathcal{E}_{m} \sin \mu_{m} x, \\
m & =1,2, \ldots
\end{aligned}
$$


for each of the corresponding eigenvalues

$$
\mu_{m}^{2}
$$

for which equation (8) is expressed as

$$
\Lambda_{m}=\kappa \mu_{m}^{2}
$$

Thus, we define $\Lambda_{k} \equiv \Lambda_{m}, U_{k} \equiv U_{m}$ and $\mathcal{E}_{k} \equiv \mathcal{E}_{m}$.

So, with this redefining the numbering we have

$$
\mathcal{L} U_{k}=\Lambda_{k} U_{k}, \quad U_{k} \in G_{\mathrm{L}}, \quad k=1,2, \ldots
$$

These eigenfunctions of $\mathcal{L}$ can be chosen orthonormal with

$$
\mathcal{E}_{k}=1 / \sqrt{\int_{0}^{a} \sin ^{2} \mu_{m_{k}} x d x}
$$

Thereby

$$
\begin{aligned}
\left\langle U_{k}, U_{l}\right\rangle & \equiv \int_{0}^{a} U_{k}(x) U_{l}(x) d x \\
& =\left\{\begin{array}{c}
\frac{\int_{0}^{a} \sin \mu_{m_{k}} x \sin \mu_{m_{l}} x d x}{\int_{0}^{a} \sin ^{2} \mu_{m_{k}} x d x}=\delta_{k l}, \\
m_{k}, m_{l}=1,2, \ldots,
\end{array}\right.
\end{aligned}
$$

where the subscripts $k, l$ of $m$ correspond to the respective eigenfunction. $\left\{U_{k}\right\}$ is a complete set of $\mathbf{L}^{2}[0, a]$ and each function $u(x) \in G_{\mathbf{L}}$ can be represented as a series

$$
u(x)=\sum_{k=1}^{\infty}\left\langle u, U_{k}\right\rangle U_{k}(x)
$$

For $t>0$, the solution of the problem of anomalous diffusion equation of heat (2) that satisfies the prescribed initial and boundary conditions can be written as

$$
W(x, t)=\sum_{k=1}^{\infty} U_{k}(x) T_{k}(t),
$$

where $T_{k}(t)=\left\langle W, U_{k}\right\rangle$. To find the fractional differential equation for functions $T_{k}(t)$, solution (12) is substituted into equation (2)

$$
\sum_{l=1}^{\infty} U_{l}(x)\left(\frac{d}{d t}\right)^{\alpha} T_{l}(t)=-\sum_{l=1}^{\infty} T_{l}(t) \cdot \mathcal{L} U_{l}(x)+F(x, t)
$$




$$
=-\sum_{l=1}^{\infty} T_{l}(t) \cdot \Lambda_{l} U_{l}(x)+F(x, t) .
$$

After taking the scalar product of this equation for the eigenfunction $U_{k}$,

$$
\sum_{l=1}^{\infty}\left\langle U_{k}, U_{l}\right\rangle\left(\frac{d}{d t}\right)^{\alpha} T_{l}(t)=-\sum_{l=1}^{\infty} T_{l}(t) \cdot \Lambda_{l}\left\langle U_{k}, U_{l}\right\rangle+\left\langle U_{k}, F\right\rangle
$$

and using the orthonormality of eigenfunctions, we obtain the equation

$$
\left(\frac{d}{d t}\right)^{\alpha} T_{k}(t)+\Lambda_{k} T_{k}(t)=f_{k}(t),
$$

with $f_{k}(t) \equiv\left\langle U_{k}, F\right\rangle, \quad k=1,2, \ldots$. Applying the initial condition (2) to the equation (12), we have

$$
\begin{gathered}
W(x, 0)=f(x)=\sum_{k=1}^{\infty} U_{k}(x) T_{k}(0), \\
T_{k}(0)=\left\langle\left. W\right|_{t=0}, U_{k}\right\rangle=\left\langle f, U_{k}\right\rangle .
\end{gathered}
$$

For the initial condition $T_{k}(0)$, note that the solution of the corresponding homogeneous problem (2) (i.e., with $F(x, t) \equiv 0$ ) has the form

$$
W_{\mathrm{H}}(x, t)=\sum_{k=1}^{\infty} U_{k}(x) T_{\mathrm{H}, k}(t),
$$

where

$$
T_{\mathrm{H}, k}(t)=A_{\mathrm{H}, k} E_{\alpha}\left(-\Lambda_{k} t^{\alpha}\right), \quad k=1,2, \ldots
$$

is the general solution of the homogeneous equation corresponding to (13) (since $f_{k}(t) \equiv 0$ if $F=0$ ) for each $\Lambda_{k}$ (Luchko and Gorenflo [4]).

Each $A_{\mathrm{H}, k}$ is an arbitrary constant which is determined by applying the homogeneous initial condition, which is the same as for the nonhomogeneous equation $\left(W_{\mathrm{H}}(x, 0)=W(x, 0)=f(x)\right)$,

$$
W_{\mathrm{H}}(x, 0)=\sum_{l=1}^{\infty} U_{l}(x) A_{\mathrm{H}, l}=W(x, 0)=f(x),
$$

from which we get by taking the dot product of $f$ and $U_{k}$ and considering (14), 


$$
T_{k}(0)=\left\langle U_{k}, f\right\rangle=A_{\mathrm{H}, k}
$$

That is, for $m_{k} \geq 1$,

$$
A_{\mathrm{H}, k}=\frac{1}{\sqrt{\int_{0}^{a} \sin ^{2} \mu_{m_{k}} x d x}} \int_{0}^{a} f(x) \sin \mu_{m_{k}} x d x .
$$

To find the solution of the Cauchy problem for equation (13) with the initial condition (16), we consider the following. By Lemma 4, we have

$$
\left(\frac{d}{d t}\right)^{\alpha} T_{k}(t)=I_{0+}^{1-\alpha} T_{k}^{\prime}(t)
$$

Substituting this result in equation (13), the following equation is obtained:

$$
I_{0+}^{1-\alpha} T_{k}^{\prime}(t)+\Lambda_{k} T_{k}(t)=f_{k}(t) .
$$

Applying the operator $I_{0+}^{\alpha}$ to this equation, we obtain the following Volterra integral equation of the second kind:

$$
T_{k}(t)=I_{0+}^{\alpha} f_{k}(t)+T_{k}(0)-\frac{\Lambda_{k}}{\Gamma(\alpha)} \int_{0}^{t}(t-\tau)^{\alpha-1} T_{k}(\tau) d \tau .
$$

According to the Theorem 5, and using the formulas (Nahushev [6] and Djrbashyan [1])

$$
\begin{gathered}
\frac{1}{\Gamma(\xi)} \int_{0}^{z} \tau^{\beta-1} E_{\alpha, \beta}\left(\zeta \tau^{\alpha}\right)(z-\tau)^{\xi-1} d \tau=z^{\beta+\xi-1} E_{\alpha, \beta+\xi}\left(\zeta z^{\alpha}\right) \\
\frac{1}{\Gamma(\beta)}+z E_{\alpha, \alpha+\beta}(z)=E_{\alpha, \beta}(z)
\end{gathered}
$$

the integral equation (17), considering the initial condition (16), has a solution $T_{k}(t)$ defined only by the following formula:

$$
T_{k}(t)=A_{\mathrm{H}, k} E_{\alpha}\left(-\Lambda_{k} t^{\alpha}\right)+\int_{0}^{t} e_{\alpha}^{-\Lambda_{k}(t-\tau)} f_{k}(\tau) d \tau
$$

Substituting this in the series (12), we obtain the formal solution of the problem given by the equation of heat subdiffusion (2) that satisfies the initial and boundary conditions given here:

$$
W(x, t)=\sum_{k=1}^{\infty} U_{k}(x)\left[A_{\mathrm{H}, k} E_{\alpha}\left(-\Lambda_{k} t^{\alpha}\right)+\int_{0}^{t} e_{\alpha}^{-\Lambda_{k}(t-\tau)} f_{k}(\tau) d \tau\right] .
$$


So the solution of the equation of anomalous subdiffusion heat that meets the prescribed boundary conditions can be written as

$$
\begin{aligned}
W(x, t)= & \sum_{m=1}^{\infty} \mathcal{A}_{m} E_{\alpha}\left(\left[-\kappa \mu_{m}^{2}\right] t^{\alpha}\right) \sin \mu_{m} x \\
& +\sum_{m=1}^{\infty} \mathcal{E}_{m} \int_{0}^{t} e_{\alpha}^{\left[-\kappa \mu_{m}^{2}\right](t-\tau)} f_{k}(\tau) d \tau \sin \mu_{m} x
\end{aligned}
$$

where functions $f_{k}(t)$ are given by (13) for subscript $m$ corresponding, coefficients $\mathcal{E}_{m}$ are given by (11), coefficients $\mathcal{A}_{m}$ are given, for $m \geq 1$, by

$$
\mathcal{A}_{m}=\sqrt{\frac{1}{\int_{0}^{a} \sin ^{2} \mu_{m} x d x}} \int_{0}^{a} f(x) \sin \mu_{m} x d x
$$

\section{References}

[1] M. M. Djrbashyan, Integral Transformations and Representation of Functions in Complex Domain, Nauka, Moscow (1966).

[2] P. DuChateau, D. Zachmann, Applied Partial Differential Equations, Dover, New York (2002).

[3] A. A. Kilbas, H. M. Srivastava, J. J. Trujillo, Theory and Applications of Fractional Differential Equations, vol. 204, Elsevier, North Holland (2006), http://dx.doi.org/10.1016/S0304-0208(06)80001-0.

[4] Yu. Luchko, R. Gorenflo, An operational method for solving fractional differential equations with the Caputo derivatives, Acta Math. Vietnam., 24, No. 2, (1999), 207-233.

[5] F. Mainardi, G. Pagnini, R. Gorenflo, Some aspects of fractional diffusion equations of single and distributed order, $A p$ plied Mathematics and Computation, 187, No. 1, (2007), 295-305, http://dx.doi.org/10.1016/j.amc.2006.08.126.

[6] A. A. Nahushev, Elements of Fractional Calculus and Their Applications, Nalchik, Russia (2010). 
